\title{
SeEding Method FOR ICE NuCleation Under Shear
}

A PREPRINT

\author{
(1) Amrita Goswami \\ Department of Chemical Engineering \\ Indian Institute of Technology Kanpur \\ amritag@iitk.ac.in \\ (1) Jayant K. Singh* \\ Department of Chemical Engineering \\ Indian Institute of Technology Kanpur \\ indrasd@iitk.ac.in \\ Indranil Saha Dalal ${ }^{*}$ \\ Department of Chemical Engineering \\ Indian Institute of Technology Kanpur \\ jayantks@iitk.ac.in
}

August 7, 2020

\begin{abstract}
Hydrodynamic flow can have complex and far-reaching consequences on the rate of homogenous nucleation. We present a general formalism for calculating the nucleation rates of simply sheared systems. We have derived an extension to the conventional Classical Nucleation Theory, explicitly embodying the shear rate. Seeded Molecular Dynamics simulations form the backbone of our approach. The framework can be used for moderate supercoolings, at which temperatures brute-force methods are practically infeasible. The competing energetic and kinetic effects of shear arise naturally from the equations. We show how the theory can be used to identify shear regimes of ice nucleation behaviour for the $\mathrm{mW}$ water model, unifying disparate trends reported in the literature. At each temperature, we define a crossover shear rate in the limit of $1000-10,000 \mathrm{~s}^{-1}$, beyond which the nucleation rate increases steadily upto a maximum, at the optimal shear rate. For 235, 240, 255 and $260 \mathrm{~K}$, the optimal shear rates are in the range of $\approx 10^{6}-10^{7} \mathrm{~s}^{-1}$. For very high shear rates beyond $10^{8} \mathrm{~s}^{-1}$, nucleation is strongly inhibited. Our results indicate that the shear-dependent nucleation rate curves have a non-monotonic dependence on temperature.
\end{abstract}

Keywords rare-event, nucleation, shear, seeding, Classical Nucleation Theory, biasing method

\section{Introduction}

Crystal formation under the action of shear flow is a ubiquitous phenomenon, with important implications in nature and various medical, metallurgical and industrial applications [1-6]. Hydrodynamic flows can induce complex changes in the behaviour of nucleating systems, ranging from shear-mediated ordering of the liquid [7-[10] to disruption of crystallization [11, 12].

The literature is rife with contradictory accounts of the effects of shear on the nucleation rate. Certain experiments surmise that shear flow can retard nucleation [13, 14], while others report that shear flows have a negligible influence on crystallization [15--17]. Different studies have shown that shear flow can enhance the rate of nucleation [18-[22]. On the other hand, simulation studies suggest that the nucleation rates have a non-monotonic dependence on the applied shear rates, and exhibit a maximum at an optimal shear rate [23-26].

Even without the added variable of shear, the use of computer simulations for describing homogenous crystal nucleation is complicated by the fact that it is a rare event, occurring on time scales inaccessible to conventional Molecular Dynamics (MD). Techniques of analyzing crystal nucleation, dependent on brute-force simulations, are typically only

\footnotetext{
* Corresponding Author
} 
feasible for deeply supercooled systems. This is partly due to the stochastic nature of nucleation events. In practice, obtaining sufficiently good statistics from brute-force MD-based methods can be intractable for moderate supercooling [27].

The event at the crux of crystal nucleation, which is an activated process, is the formation of a sufficiently large crystalline cluster in the metastable liquid phase to overcome the free-energy barrier. Seeded MD simulations can be used to reliably estimate the critical nucleus size and the interfacial energy [28-30]. In the quintessential seeding method, the information gleaned from seeded simulations is used in conjunction with Classical Nucleation Theory (CNT) [31, 32] to calculate the free-energy barrier and nucleation rate. Thus, the seeding technique can be particularly advantageous for moderate supercoolings, at which temperatures the spatiotemporal resolution of brute-force MD can be insufficient to observe nucleation events.

In this work, we extend CNT to incorporate shear, and propose a general, computationally efficient framework that builds on the seeding method. We study the effect of a wide range of shear rates on the ice nucleation rates at different temperatures, for which brute-force MD and equilibrium rare-event sampling methods are essentially infeasible. We show that the formalism can pinpoint distinct regimes of ice nucleation behaviour, and is thus able to consolidate and justify the seemingly inconsistent results reported in the literature. The results suggest a previously unexplored non-linear temperature dependence of the nucleation rates.

\section{Theory and Methods}

\subsection{Overview of the Seeding Method}

According to Classical Nucleation Theory (CNT) [27, 31-33], the free energy of formation $F$ of a spherical crystallite nucleus of radius $R$ can be expressed as the sum of a favourable volume term and an unfavourable surface term:

$$
F(R)=-\frac{4}{3} \pi R^{3} \frac{|\Delta \mu|}{v^{\prime}}+4 \pi R^{2} \sigma,
$$

where $|\Delta \mu|$ is the chemical potential difference between the metastable liquid phase and the crystal, $v^{\prime}$ is the volume of one particle or molecule in the crystal, and $\sigma$ is the crystal-fluid interfacial free energy.

In the seeding method [34, 35], a solid cluster of the crystal phase is inserted into the supercooled fluid, and the temperature at which the cluster has a critical number of molecules $\left(N^{*}\right)$ is determined. For smaller clusters, the free energy cost of forming the solid-liquid interface dominates and the embryos tend to shrink. For clusters larger than the critical nucleus size, the volume contribution is greater, favouring crystal growth.

The critical cluster size $N^{*}$ is the cluster size which maximizes the free energy in Eq.(1), given by

$$
N^{*}=\frac{32 \pi v^{\prime 2} \sigma^{3}}{3|\Delta \mu|^{3}}
$$

where $N^{*}$ is the number of particles or molecules in the critical cluster. The critical cluster corresponds to a particular temperature, at which roughly half of the trajectories show crystal growth. This implies that, for a known inserted cluster size, the temperature at which the cluster is critical can be estimated.

Thus, the seeding technique directly yields $N^{*}$ at the estimated temperature $T$. Once $T$ has been fixed, $|\Delta \mu|$ and $v^{\prime}$ become fixed as well. The interfacial energy $\sigma$, can now be determined from Eq. (2) as:

$$
\sigma=\left(\frac{3 N^{*}}{32 \pi v^{\prime 2}}\right)^{\frac{1}{3}}|\Delta \mu| .
$$

Putting Eq. (2) in Eq. (1), we obtain the free energy barrier for nucleation:

$$
F\left(N^{*}\right)=\frac{16 \pi v^{\prime 2} \sigma^{3}}{3|\Delta \mu|^{2}}
$$

where $F\left(N^{*}\right)$ is the height of the free energy barrier. 
The rate of nucleation can be estimated as $[36-39]$

$$
J=\rho_{l} Z f^{+} e^{-\frac{F\left(N^{*}\right)}{k_{B} T}}
$$

where $\left(\rho_{l} Z f^{+}\right)$is the kinetic pre-factor, in which $\rho_{l}$ is the number density of the supercooled liquid, $Z$ is the Zeldovich factor [37], and $f^{+}$is the rate of attachment of molecules to the cluster in units of inverse time. $k_{B}$ is the Boltzmann constant, and $T$ is the temperature at which the inserted seed of size $N^{*}$ is critical.

The Zeldovich factor $Z$ captures the multiple possible re-crossings of the free energy barrier [40] and is related to the curvature of the free energy curve at the critical cluster size $N^{*}$. According to CNT, which assumes a perfectly spherical cluster, the Zeldovich factor can be approximated by:

$$
Z=\sqrt{\frac{\left|F^{\prime \prime}\left(N^{*}\right)\right|}{2 \pi k_{B} T}}=\sqrt{\frac{|\Delta \mu|}{6 \pi k_{B} T N^{*}}}
$$

where $F^{\prime \prime}\left(N^{*}\right)=\left.\frac{\partial^{2} F(N)}{\partial N^{2}}\right|_{N=N^{*}}$.

Thus, $Z$ can be directly calculated using Eq. 6 from the quantities obtained from seeding computations and the chemical potential difference between the liquid and solid phases $|\Delta \mu|$.

The main advantage of the seeding technique is that it allows for a direct estimate of the interfacial free energy for a wide range of supercooling conditions. Using the framework of CNT, the free energy barrier and the nucleation rate can be calculated from quantities obtained from seeded simulations, the chemical potential difference and the crystal density.

In the subsequent sections, we extend the CNT equations for bulk homogenous systems to account for volume-preserving shear, building on the seeding method.

\subsection{Extension of the Seeding Technique and CNT for Sheared Systems}

According to Mura and Zaccone [25], the free energy of a crystal nucleus in a bulk nucleating system, subjected to a volume-preserving shear ("simple" shear) $\dot{\gamma}$, is given by

$$
F(R)=-\frac{4}{3} \pi R^{3} \frac{\left|\Delta \mu_{0}\right|}{v^{\prime}}+4 \pi R^{2} \sigma_{0}\left(1+\frac{7}{24} \frac{\eta^{2} \dot{\gamma}^{2}}{G^{2}}\right)+\frac{1}{2} \frac{\eta^{2} \dot{\gamma}^{2}}{G} \frac{4}{3} \pi R^{3}
$$

where $F(R)$ is the free energy of formation of a cluster of radius $R,\left|\Delta \mu_{0}\right|$ is the chemical potential difference between the thermodynamically stable crystal phase and the metastable liquid phase when no shear is applied, $\sigma_{0}$ is the surface tension or the interfacial free energy of the nucleus at zero shear, $v^{\prime}$ is the volume of one molecule in the crystal phase, $\eta$ is the fluid viscosity, and $G$ is the shear modulus of the nucleus.

Here, the term $\frac{7}{24} \frac{\eta^{2} \dot{\gamma}^{2}}{G^{2}}$ in Eq.(7) is a "shape factor" correction; which accounts for the deformation of the nucleus into an ellipsoid.

As noted in the previous section, the eponymous seeding technique provides a direct estimate for the interfacial free energy $\sigma_{0}$, at the temperature $T$ for which the inserted seed is critical. Thus, it is desirable to convert the free energy in Eq. (7) to a function of the cluster size $N$. Changing the variable $R$ to $N$ enables us to rewrite the free energy as

$$
F(N)=-N\left(\frac{2 G\left|\Delta \mu_{0}\right|-\eta^{2} \dot{\gamma}^{2} v^{\prime}}{2 G}\right)+\frac{\pi \sigma_{0}}{6 G^{2}}\left(\frac{3 v^{\prime} N}{4 \pi}\right)^{\frac{2}{3}}\left(24 G^{2}+7 \eta^{2} \dot{\gamma}^{2}\right)
$$

The equation above has a stationary point corresponding to the critical nucleus size $N^{*}$. Solving for the stationary point of Eq.8], we obtain:

$$
N^{*}=\frac{\pi \sigma_{0}^{3} v^{\prime 2}}{162 G^{3}}\left(\frac{24 G^{2}+7 \eta^{2} \dot{\gamma}^{2}}{2 G\left|\Delta \mu_{0}\right|-\eta^{2} \dot{\gamma}^{2} v^{\prime}}\right)^{3}
$$


The critical nucleus size corresponds to the stationary point of the free energy surface, defined by Eq. 88 ). Thus the height of the free energy barrier for nucleation, corresponding to the critical nucleus size $N^{*}$, is given by

$$
F\left(N^{*}\right)=\frac{\pi \sigma_{0}^{3} v^{\prime 2}}{648 G^{4}} \frac{\left(24 G^{2}+7 \eta^{2} \dot{\gamma}^{2}\right)^{3}}{\left(2 G\left|\Delta \mu_{0}\right|-\eta^{2} \dot{\gamma}^{2} v^{\prime}\right)^{2}} .
$$

We note that the free energy barrier increases with an increase in the shear rate as governed by Eq.(10). By construction, Eq. (9) also predicts a concomitant increase in $N^{*}$.

\subsection{Calculation of the Nucleation Rate}

The dynamics of a nucleation process can be formulated as a Kramers-Moyal expansion [41, 42], which can be recast as a quintessential Focker-Planck equation [43]

$$
\frac{\partial P(N, t)}{\partial t}=\frac{\partial}{\partial N}\left(D e^{-\frac{F(N)}{k_{B} T}} \frac{\partial}{\partial N}(P(N, t)) e^{\frac{F(N)}{k_{B} T}}\right),
$$

where $P(N, t)$ is the probability distribution of the clusters containing $N$ molecules at time $t$, and $D$ is a diffusion coefficient, or in this case, the rate of attachment of molecules to a nucleus in units of inverse time. $F(N)$ (Eq. (8)) is the free energy barrier for this nucleation process.

We recognize that Eq. (11) can be reformulated as the Zeldovich-Frenkel equation [36, 37]

$$
J=-D e^{-\frac{F(N)}{k_{B} T}} \frac{\partial}{\partial N}\left(P(N, t) e^{\frac{F(N)}{k_{B} T}}\right),
$$

where $J$ is the current or flux across the free energy barrier, in the cluster-size space.

The solution to the system of equations above yields the familiar CNT form of the steady-state nucleation rate [36-39]

$$
J=\rho_{l} Z f^{+} e^{-\frac{F\left(N^{*}\right)}{k_{B} T}}
$$

where the nucleation rate $J$ is in units of the number of nucleation events per unit volume per unit time, $f^{+}$is the attachment rate of molecules to the critical cluster, $\rho_{l}$ is the number density of the supercooled liquid, and $Z$ is the Zeldovich factor.

The Zeldovich factor is a measure of the curvature at the top of the free energy barrier. $Z$ accounts for the fact that postcritical clusters might still shrink without growing due to re-crossing the barrier [40]. According to our formalism, as expressed in the cluster-size-space, $Z$ can be calculated using:

$$
Z=\sqrt{\frac{\left|F^{\prime \prime}\left(N^{*}\right)\right|}{2 \pi k_{B} T}}=\left(\frac{v^{\prime}}{36 \pi N^{* 2}}\right)^{\frac{1}{3}} \sqrt{\frac{\sigma_{0}\left(24 G^{2}+7 \eta^{2} \dot{\gamma}^{2}\right)}{6 G^{2} k_{B} T}} .
$$

Thus we have derived expressions for the height of the free energy barrier $F\left(N^{*}\right)$, and the steady-state nucleation rate $J$ in terms of the shear rate, and equilibrium properties $\left|\Delta \mu_{0}\right|$ and $\sigma_{0}$ determined in the absence of shear. Later sections show that these calculations can be performed in a computationally efficient framework. The other quantities required for the calculation of $J$ are $f^{+}, v^{\prime}, \eta$ and $G$. These input parameters can either be obtained directly from the seeding technique or estimated otherwise.

\subsection{Calculation of the Attachment Rate}

According to CNT, the attachment rate $f^{+}$can be related to the time required for a single particle or molecule to attach to the crystal cluster [36, 37]. We can estimate $f^{+}$by

$$
f^{+}=\frac{6 D_{l}}{\lambda^{2}} O_{N^{*}},
$$


where $D_{l}$ is the diffusion coefficent of the supercooled liquid phase, $\lambda$ is the atomic 'jump length', estimated to be about one molecule diameter, and $O_{N^{*}}$ is the number of binding sites on the surface of the critical cluster of size $N^{*}$. $O_{N^{*}}$ can be assumed to be equal to the surface area of the cluster, divided by an area per molecule or particle [36]. The per-particle area in contact with the cluster is assumed to be $\pi\left(\frac{3 v^{\prime}}{4 \pi}\right)^{\frac{2}{3}}[36]$. Hence, we obtain:

$$
O_{N^{*}}=4\left(N^{*}\right)^{\frac{2}{3}}\left(1+\frac{7 \eta^{2} \dot{\gamma}^{2}}{24 G^{2}}\right)
$$

Substituting the value of $O_{N^{*}}$ from Eq.(16) and putting it in Eq.(15), the final expression for $f^{+}$is

$$
f^{+}=\frac{24 D_{l}}{\lambda^{2}}\left(N^{*}\right)^{\frac{2}{3}}\left(1+\frac{7 \eta^{2} \dot{\gamma}^{2}}{24 G^{2}}\right)
$$

We recall that the term $\frac{7 \eta^{2} \dot{\gamma}^{2}}{24 G^{2}}$ is a "shape factor" accounting for the deformation of the cluster due to the application of shear. For approximately spherical crystallites, $\frac{7 \eta^{2} \dot{\gamma}^{2}}{24 G^{2}}$ is negligible. However, the application of shear still has the overall effect of enhancing $f^{+}$since both $D_{l}$ and $N^{*}$ increase with increasing shear rates.

\subsubsection{Calculation of the Diffusion Coefficient}

We expect the diffusion coefficient $D_{l}$ to be dependent on the shear rate [25, 26, 44, 45], in addition to being dependent on the temperature [46]. In our simulations, the applied shear rate is in the $x$ dimension. Hence, we can calculate the two-dimensional diffusion coefficient $\left(D_{l}\right)$ of the supercooled liquid under the application of shear, in the $y z$ plane using the mean square displacement [47]

$$
D_{l}=\lim _{t \rightarrow \infty} \frac{1}{4 t}\left\langle[y(t)-y(0)]^{2}+[z(t)-z(0)]^{2}\right\rangle,
$$

where $D_{l}$ is the two-dimensional self-diffusion coefficient, $t$ is the elapsed time with respect to a reference time origin, $\left\langle[y(t)-y(0)]^{2}+[z(t)-z(0)]^{2}\right\rangle$ is the ensemble average over all molecules and time origins, of the mean-squared displacement of the molecules in the $y$ and $z$ dimensions.

\subsection{Limiting Values of Shear Rates}

The denominator of the free energy in Eq. 10 is undefined for larger shear rates as it tends to shrink to zero. The correspondingly infinite free energy barrier has well-defined physical consequences. This situation implies that the clusters are not mechanically stable at very large shear rates, and this effectively suppresses nucleation. We define the limiting maximum shear rate $\dot{\gamma}_{\max }$ as the shear rate for which the free energy barrier is infinite and for which the nucleation rate effectively vanishes, given by

$$
\dot{\gamma}_{\text {max }}=\frac{1}{\eta}\left(\frac{2 G\left|\Delta \mu_{0}\right|}{v^{\prime}}\right)^{\frac{1}{2}},
$$

where $G$ is the shear modulus in units of pressure, $\left|\Delta \mu_{0}\right|$ is the difference in chemical potential between the liquid and solid phases in the absence of shear, $\eta$ is the viscosity of the supercooled liquid, and $v^{\prime}$ is the volume of a single molecule in the crystal phase. $v^{\prime}$ is, hence, the reciprocal of the number density of the solid phase.

We note the dependence of $\dot{\gamma}_{\max }$ on the mechanical properties of the crystal nucleus ( $G$ and $\eta$ ), as well as on the chemical potential difference and the density of the crystal seed.

Interestingly, $\dot{\gamma}_{\max }$ in Eq. $\left[19\right.$ ) is not dependent on the value of $N^{*}$, which implies that for values of shear rates greater than $\dot{\gamma}_{\max }$ even infinitesmally small nuclei are mechanically unstable and prone to fragmentation.

Rigorous calculations at every temperature yield the values of $\left|\Delta \mu_{0}\right|$ and $\eta$ at each temperature [28, 29. 48]. However, in order to determine the dependence of $\dot{\gamma}_{\max }$ on the temperature $T$, it is desirable to approximate both $\left|\Delta \mu_{0}\right|$ and $\eta$ by analytical functions of $T$. Using the enthalpy change of melting [37] and a power law expression for viscosity [48], we can rewrite Eq. 19] in terms of $T$ by using such analytical approximations for $\left|\Delta \mu_{0}\right|$ and $\eta$, respectively. 
The power law expression for $\eta$ for supercooled water is given by Dehaoui et al. [48]

$$
\eta=A_{0}\left(\frac{T}{T_{S}}-1\right)^{-\beta}
$$

where $A_{0}$ and $T_{S}$ are fitting parameters with units of dynamic viscosity and temperature, respectively. $\beta$ is a dimensionless fitting parameter.

The difference in the chemical potential, $\Delta \mu_{0}$, can be approximated using the enthalpy change at melting [37], which is accurate for low supercoolings and specifically for the monoatomic $(\mathrm{mW})$ water model [49] at larger supercoolings can be given by:

$$
\Delta \mu_{0}=\Delta H_{m}\left(1-\frac{T}{T_{m}}\right),
$$

where $\Delta H_{m}$ is the enthalpy change at melting, and $T_{m}$ is the melting point for the model.

Eliminating $\eta$ and $\left|\Delta \mu_{0}\right|$ from Eq. (19), using Eq.20) and Eq.(21), respectively, we recast $\dot{\gamma}_{\max }$ as a function of $T$ :

$$
\dot{\gamma}_{\max }(T)=\frac{\left(T-T_{S}\right)^{\beta}}{A_{0} T_{S}^{\beta}}\left(\frac{2 G}{v^{\prime}} \Delta H_{m}\left(1-\frac{T}{T_{m}}\right)\right)^{\frac{1}{2}} .
$$

We can estimate the validity of the approximations made, by comparing the values of $\dot{\gamma}_{\max }$ calculated using explicitly determined $\eta$ and $\left|\Delta \mu_{0}\right|$ values from the literature [28, 29, 48], with those predicted by Eq.222.

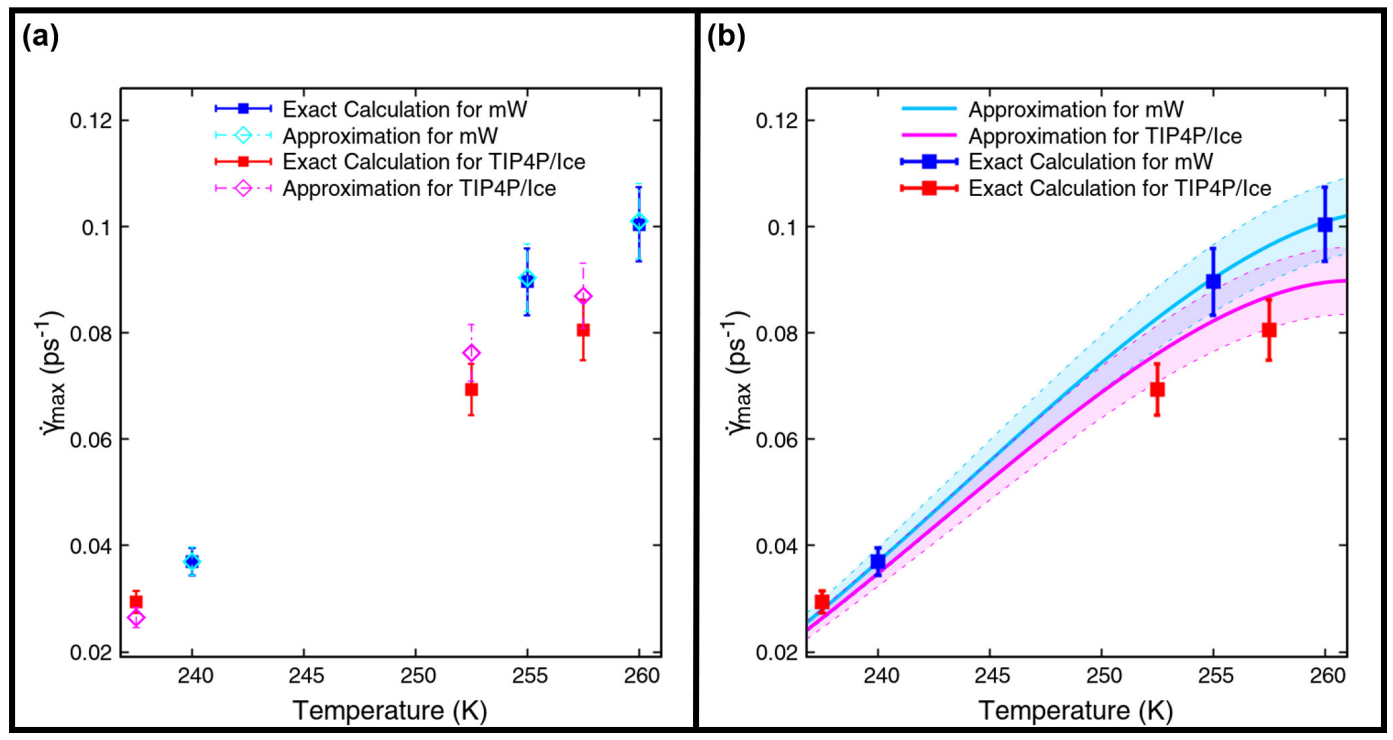

Figure 1: (a) Maximum limiting values of the shear rate plotted against temperature, calculated using explicitly determined $\eta$ and $\left|\Delta \mu_{0}\right|$ values for the $\mathrm{mW}$ (filled blue squares) and TIP4P/Ice (filled red squares) water models [28, 29. 48]. Calculations with approximated $\dot{\gamma}_{\max }$ estimated from Eq. (22) for $\mathrm{mW}$ and TIP4P/Ice are shown as open diamond symbols in turquioise and magenta, respectively. (b) Comparison of the more exact estimations of $\dot{\gamma}_{\max }$ with analytical expressions (Eq. 222) using a constant ice density for each water model. The two dotted lines sandwiching the functional approximations for the $\mathrm{mW}$ (solid turquoise line) and TIP4P/Ice (solid magenta line) models show the uncertainty (1 standard deviation).

Figure 1 (a) shows the trend of $\dot{\gamma}_{\max }$ with temperature for the mW and TIP4P/Ice [50] water models. Evidently, the the approximate relation in Eq. 22 agrees well with the more exact calculations of $\dot{\gamma}_{\max }$ for the $\mathrm{mW}$ model, as expected. Figure 1 a) shows that, within the statistical uncertainty, the values of $\dot{\gamma}_{\max }$ for the mW model nearly coincide. However, there is more divergence of the approximate values for the TIP4P/Ice model. 
It has been observed that the density of hexagonal ice (Ih) increases with decreasing temperature at constant pressure [51. 52], which is a trend also reflected in experiments [53]. However, we can further approximate the value of $v^{\prime}$ in Eq. (22) by assuming that the Ih density is constant in the temperature range $235-260 \mathrm{~K}$. Figure 1 (b) depicts the change in the $\dot{\gamma}_{\max }$ with temperature, using this additional approximation. The agreement of the mW model with the predicted approximate values is still excellent. In the case of the TIP4P/Ice model, the predicted values still differ from the more exact calculations, but not significantly more than in Figure 1 (a).

We surmise that Eq.(22) can be safely used for the coarse-grained $\mathrm{mW}$ water model, but can show small divergences from more rigorous calculations, for the TIP4P/Ice model. The approximation can be used to predict general trends and behaviour with tolerable agreement for TIP4P/Ice.

\subsection{Methodology Sequence}

From Eq.(13), the quantities required for the estimation of the nucleation rate $J$ are the chemical potential difference between the stable and metastable phases $\left(\left|\Delta \mu_{0}\right|\right)$, the rate of attachment of molecules to the critical cluster $\left(f^{+}\right)$, the volume of a single molecule in the crystal phase at the thermodynamic conditions $\left(v^{\prime}\right)$, the viscosity of the supercooled liquid $(\eta)$ and the shear modulus of the crystal nucleus $(G)$. We outline the estimation of the input parameters required for the calculation of $J$, at different shear rates:

1. We employ the seeding method to estimate the temperature for known critical cluster sizes. Once a trial temperature has been fixed for a pre-determined critical cluster size, the interfacial energy $\sigma_{0}$ can be calculated. In the seeding technique, spherical clusters of pure Ih of a certain size are inserted into equilibrated configurations of supercooled liquid [28-30]. For a given inserted cluster seed size, the temperature $T$ for which the seed is critical can be determined by running several trajectories and tracking the cluster sizes. If the inserted cluster is of critical size at the test temperature then the seed grows in roughly half of the trajectories, while the cluster shrinks during the course of the other runs. The accuracy of this estimation of $T$ can be improved by increasing the number of runs at each trial temperature. Systematic errors are larger for smaller cluster sizes owing to the uncertainty in the determination of the interfacial region.

2. $\left|\Delta \mu_{0}\right|$ can be determined directly by using the Einstein molecule method [54] to compute the chemical potential of the fluid and crystal phases [55], or by thermodynamic integration [28]. Alternatively, Eq. 221] can be used as an approximation for $\left|\Delta \mu_{0}\right|$. As noted previously, Eq.21) is accurate only for low supercoolings and differs from rigorously calculated $\left|\Delta \mu_{0}\right|$ for atomistic water potentials [28]. However, it has been reported that Eq. [21] performs well for larger supercoolings as well [28], in the case of the $\mathrm{mW}$ water model, since the maximum of heat capacity is displaced to lower temperatures for this model [49].

3. $f^{+}$is the rate of attachment of molecules to the critical cluster, which can be calculated using Eq.(17). A typical attachment or 'jump' length $\lambda$ can be assumed to be one molecular diameter [37]. For the mW model, we can take $\lambda=3.5 \AA[28]$. The diffusion coefficient $D_{l}$, which may be dependent on shear, is also required for the calculation of $f^{+}$.

4. $v^{\prime}$ is the inverse of the number density of the crystal phase. It is known that Ih density varies with the temperature, though the extent of this density change varies for different water models [28]. The Ih density at every temperature can be determined from isothermal-isobaric simulations of the crystal phase.

5. $\eta$ is dependent on temperature, and can either be calculated directly from Molecular Dynamics simulations [56] or estimated by a power law relation [48]. We note that such analytical relations for the viscosity are typically not valid for deeply supercooled water below $230 \mathrm{~K}$. We can assume that $\eta$ is independent of the applied shear rate for 'low' shear rates; however, at higher shear rates $\eta$ decreases under shear strain. Shear-thinning has been observed for the SPC/E water model for shear rates greater than $0.1 \mathrm{ps}^{-1}$ [57]. In this work, the highest limiting shear rate calculated using Eq.(22) is $\approx 0.1 \mathrm{ps}^{-1}$, and hence we can assume that $\eta$ is constant with respect to the shear rate, at each $T$, within this limit.

6. The shear modulus $G$ can vary, in the range of $\approx 3-4.5 G P a$, for different crystal plane directions and cluster structure, for both Ih and amorphous ices $[58-60]$. Figure S2 shows the temperature dependence of $\dot{\gamma}_{\text {max }}$, estimated using Eq. 22 ) for the $\mathrm{mW}$ water model, for different values of $G$. In this work, we have used a constant shear modulus, without taking into account the anisotropy of $G$. 


\section{Simulation Details}

We have applied our formalism to the monatomic $(\mathrm{mW})$ water model [49], at four temperatures 235, 240, 255 and $260 K$.

\subsection{Seeding Method Calculations}

The seeding method was employed to obtain input parameters, described in further detail in the Supporting Information. The technique involves the generation of molecular dynamics trajectories in the absence of shear. Molecular dynamics simulations of the $\mathrm{mW}$ water model were performed using LAMMPS [61] in the isothermal-isobaric $(N P T)$ ensemble at $1 \mathrm{~atm}$. The Nose-Hoover thermostat and barostat were used to control the temperature and pressure, respectively. A time step of $10 \mathrm{fs}$ was used.

Initial systems were created by inserting a perfectly spherical cluster of hexagonal ice (Ih) in supercooled liquid configurations obtained from independent simulations at $208 \mathrm{~K}$. The liquid molecules which overlapped with the inserted cluster molecules were removed, and a tolerance of $2 \AA$ was kept between the cluster and surrounding supercooled liquid. The systems were then equilibrated in two steps. First, the solid cluster molecules were kept rigid, allowing the liquid molecules to move at a temperature of $200 \mathrm{~K}$ for $8 \mathrm{ps}$. Next, the constraint on the cluster molecules was removed and the systems were equilibrated for a further $0.2 \mathrm{~ns}$ at $200 \mathrm{~K}$.

The temperature at which each cluster is critical was determined by running MD trajectories at different temperatures and tracking the cluster size. The cluster size evolution for a seed size of 686 particles has been shown in Figure S1. The ice-like molecules were differentiated from the liquid phase by using the structural identification method of Maras et al. [62], in OVITO [63]. The evolution of the largest ice cluster in each trajectory was monitored using d-SEAMS [64].

Table S1 lists the input parameters, used in our formalism, for the $\mathrm{mW}$ water model.

\subsection{Simulations for Calculating the Diffusion Coefficient}

Molecular dynamics trajectories were obtained at different shear rates using LAMMPS [61] at 235, 240, 255 and $260 \mathrm{~K}$. The two-dimensional diffusion coefficients at different shear rates were calculated using the VMD Diffusion Coefficient Tool [65]. Shear was imposed in the $x$ dimension using the SLLOD algorithm [66], with Lees-Edwards boundary conditions [67, 68]. A timestep of $5 f s$ was used for the simulations with shear. The system size used for the diffusion coefficient calculations was 4096 molecules of $\mathrm{mW}$ water at the four temperatures considered in this work.

\section{Results and Discussion}

\subsection{Effect of Shear on Diffusion}

We observe that diffusion is enhanced by the applied shear, as expected from previous results in the literature [25. 26. 44, 45]. By fitting the two-dimensional bulk diffusion coefficients at different shear rates, we obtain the following linear relationship at a constant temperature $T$ :

$$
D_{l}=D_{0}+c \dot{\gamma}
$$

where $D_{l}$ is the two-dimensional diffusion coefficient at a particular shear rate $\dot{\gamma}$ and temperature $T, D_{0}$ is the bulk diffusion coefficient in the absence of shear at $T$, and $c$ is a fitting constant corresponding to the rate of increase of the diffusion coefficient with shear, which has units of squared length.

Figure S3 shows the linear fits of the diffusion coefficient with shear rates, for the four temperatures studied in this work. 


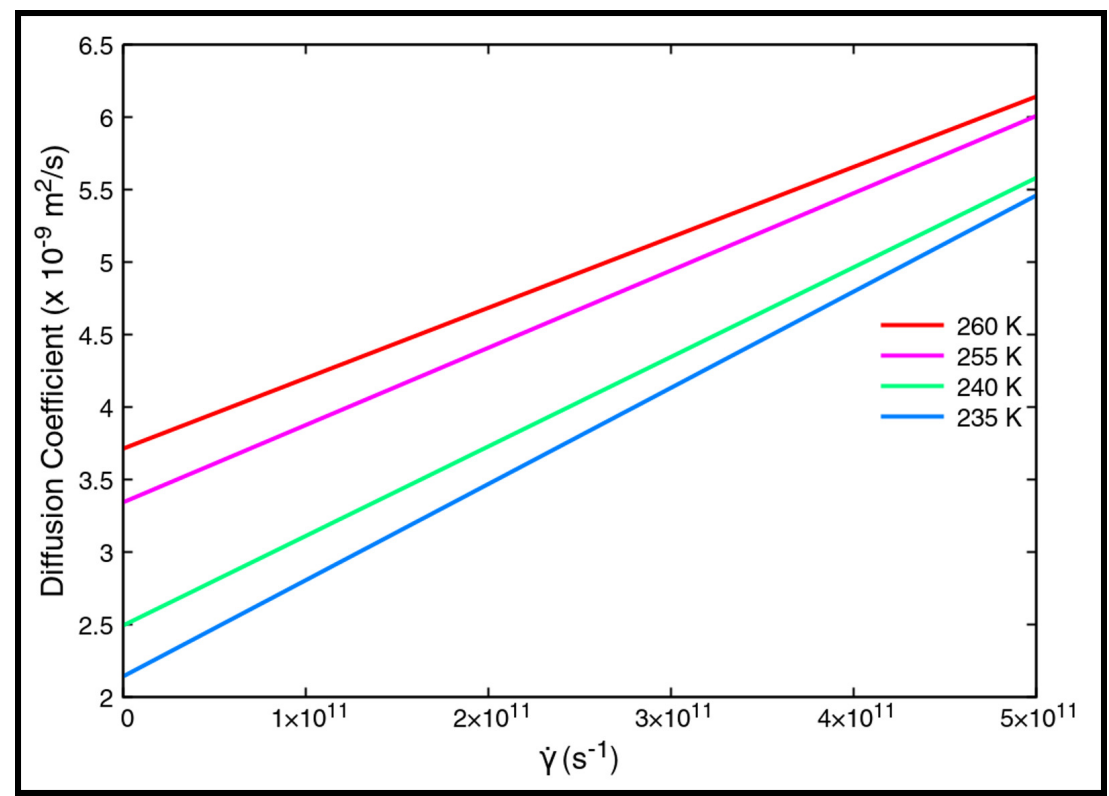

Figure 2: Variation of the Diffusion coefficient with shear, for four different temperatures. It is observed that the Diffusion coefficients increase linearly with shear.

Figure 2 depicts the fitted lines for each temperature. We observe that, in general, the diffusion coefficient increases with increasing temperature, as expected. We also surmise that the rate of increase of the diffusion coefficient $c$ decreases with increasing temperature. Table 1 lists the values of $D_{0}$ and $m$ for each temperature.

Table 1: Parameter values in $D_{l}=D_{0}+c \dot{\gamma}$

\begin{tabular}{lll}
\hline Temperature & $D_{0}\left(\times 10^{-9} \mathrm{~m}^{2} / \mathrm{s}\right)$ & $\mathrm{c}\left(\times 10^{-21} \mathrm{~m}^{2}\right)$ \\
\hline $260 \mathrm{~K}$ & 3.715 & 4.85364 \\
$255 \mathrm{~K}$ & 3.345 & 5.32611 \\
$240 \mathrm{~K}$ & 2.496 & 6.17091 \\
$235 \mathrm{~K}$ & 2.145 & 6.63036 \\
\hline
\end{tabular}

\subsection{Variation of the Nucleation Rate with Shear Rate}

The application of shear has two opposing contributions to the nucleation rate: the free energy barrier increases with the shear rate, tending to retard nucleation, while the kinetic pre-factor rises with shear. The increase in the kinetic pre-factor tends to promote nucleation. We also note that the the critical nucleus size also increases with the shear rate [23, 25], shown in Figure S4. 

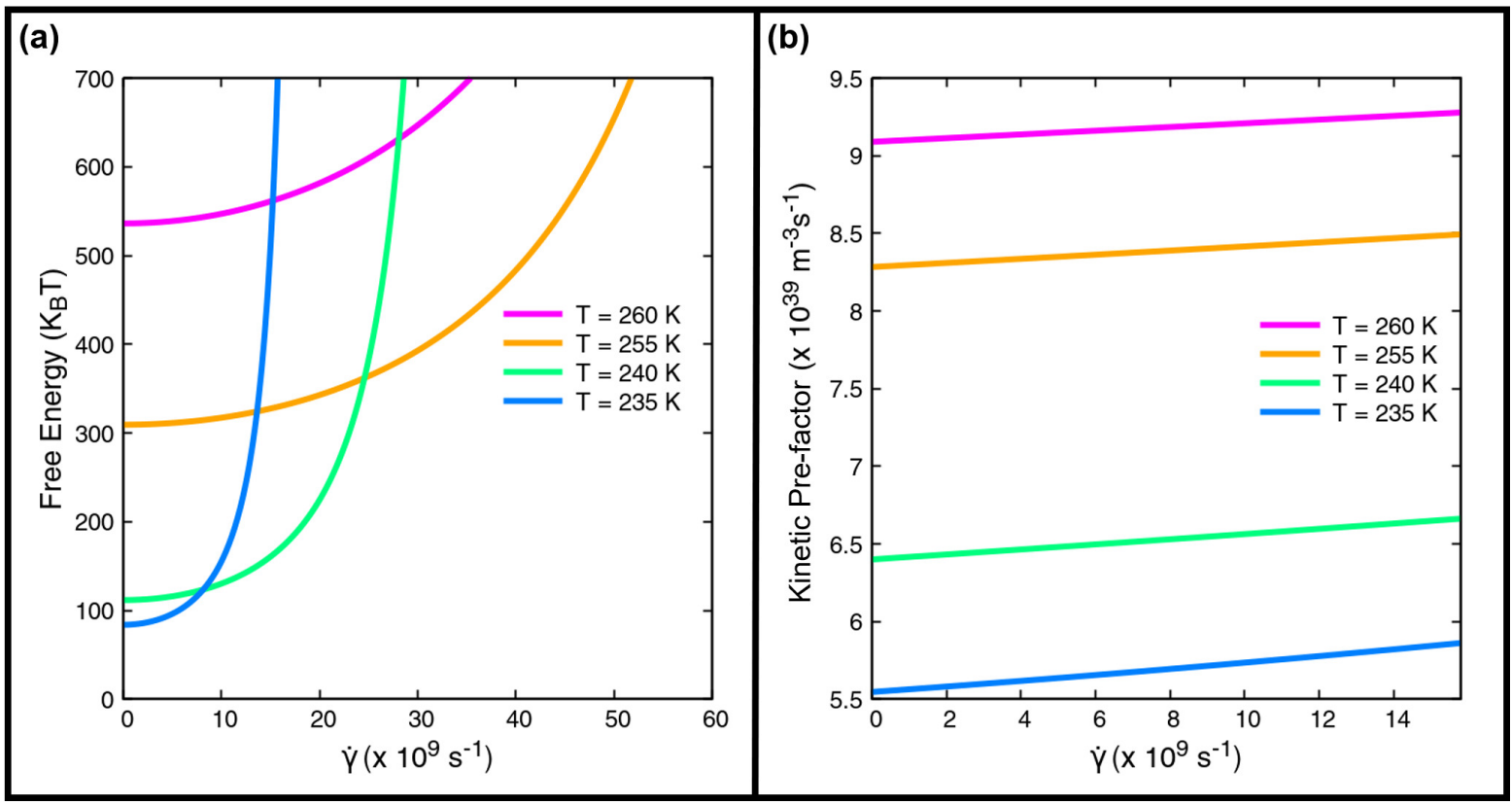

Figure 3: (a) Variation of the free energy barrier $F\left(N^{*}\right)$ with the shear rate $\dot{\gamma}$ for $260,255,240$ and $235 \mathrm{~K}$. (b) The kinetic pre-factor $\rho_{l} Z f^{+}$plotted against the shear rate $\dot{\gamma}$ for different temperatures.

Figure 3 a) shows the quadratic increase of the free energy barrier with rising shear rates. The quadratic increase in the free energy barrier of nucleation with shear has also been reported for colloidal suspensions [13]. Figure 3 (b) depicts the increase of the kinetic pre-factor with shear for different temperatures. The diffusion coefficients were calculated using Eq. 23 We surmise that the non-monotonicity of the nucleation rate arises from the interplay of these two conflicting tendencies.
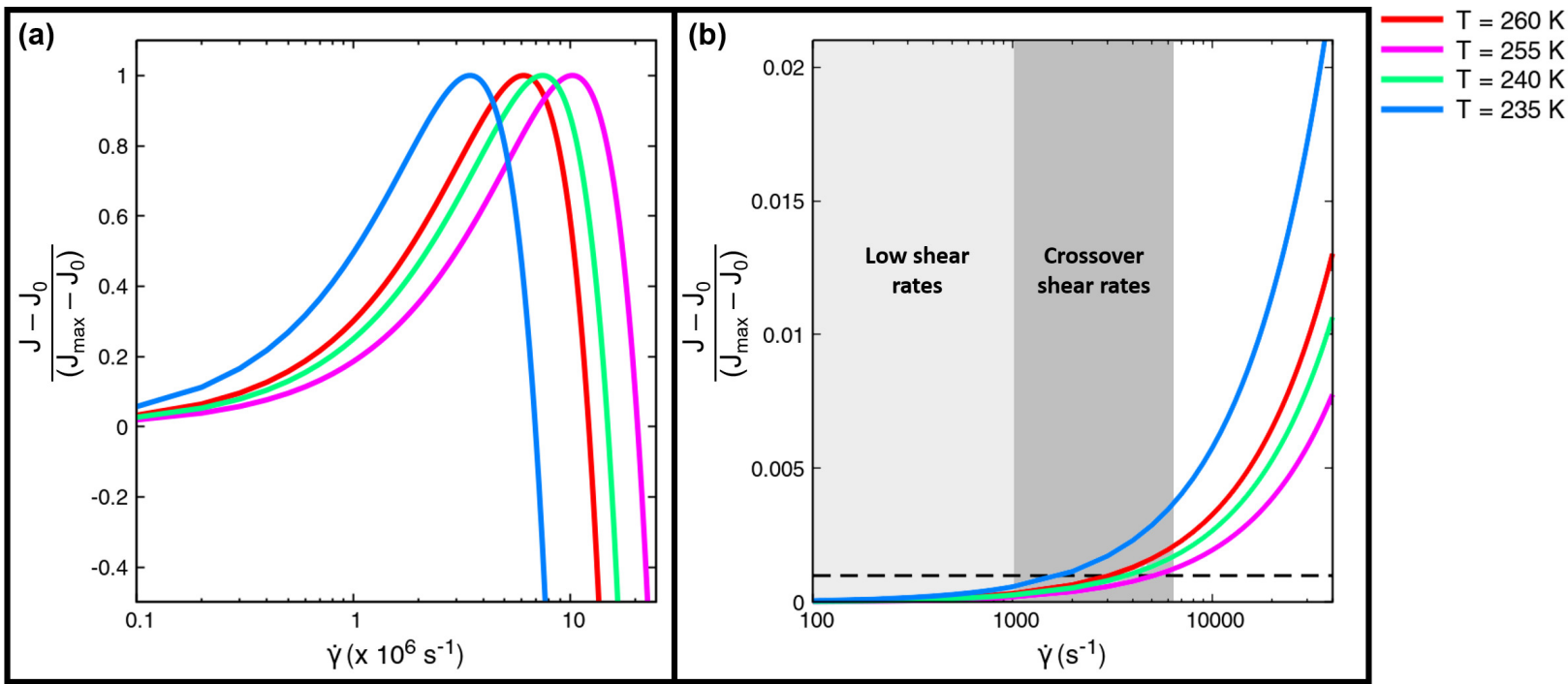

Figure 4: (a) Variation of the normalized relative nucleation rates $\frac{J-J_{0}}{J_{\max }-J_{0}}$ with shear, for shear rates greater than $10^{5} \mathrm{~s}^{-1}$, for different temperatures. The non-monotonic behaviour arises from the competing energetic and kinetic effects of shear. (b) Normalized relative nucleation rates, plotted against the shear rate, depicting the regimes of low shear rates and crossover shear rates. In the low shear rate regime, upto $1000 \mathrm{~s}^{-1}$, highlighted in light grey, the nucleation rates are unaffected by the applied shear. The nucleation rates begin to increase for shear rates greater than the crossover shear rates, shaded in dark grey. The black dotted line denotes the normalized relative nucleation rate value of 0.001 , for which the crossover shear rates are defined. 
Figure 4 (a) depicts the non-monotonicity of the dimensionless relative nucleation rates $\frac{J-J_{0}}{J_{\max }-J_{0}}$ with shear, for four different temperatures. This dimensionless relative nucleation rate is defined with respect to $J_{0}$, the nucleation rate calculated when there is no shear, and $J_{\max }$. $J_{\max }$ is the highest nucleation rate, maximized with respect to the shear rate, at a particular temperature. Figure S5 shows $J_{\max }$ obtained for the four temperatures considered in this work. Figure 4(b) shows plots of the normalized relative nucleation rate, in the limit of low shear rates.

Our results indicate the existence of three qualitative regimes of crystallization behaviour under shear. At low shear rates, in the range of $0-1000 \mathrm{~s}^{-1}$, there is negligible effect on the kinetics (highlighted in light grey in Figure 4 (b)). Such shear rates are typically imposed in Couette flows in experiments [69].

However, for shear rates between $1000-10,000 s^{-1}$, the nucleation rates begin to increase smoothly. We define a crossover shear rate, indicative of this smooth transition, such that at the crossover shear rate the normalized relative nucleation rate is 0.001 . The region of crossover shear rates for different temperatures is highlighted in dark grey in Figure 4(b).

The nucleation rate continues to rise steadily for higher shear rates for every temperature, upto shear rates of the order of $10^{6} s^{-1}$. Between $\approx 10^{6}-10^{7} s^{-1}$, a maximum in the nucleation rate is observed, originating from the competing kinetic and energetic effects of shear (Figure 4(a)). We define the shear rate at which the nucleation rate is maximum as the optimal shear rate, at a particular temperature. Beyond the optimal shear rate, very high shear rates inhibit nucleation, until the nucleation rate vanishes. In the regime of very high shear rates larger than the optimal shear rate, the free energy cost of nucleation dominates the enhancement in diffusion due to the shear.

We also note that the optimal shear rates observed are much higher than shear rates typically used in experiments. The range of shear rates generated in experiments can be about $0-100,000 s^{-1}$ [70], orders of magnitude lower than the optimal shear rates.

\subsection{Dependence of the Optimal Shear Rates and Nucleation Rate Curves on Temperature}

The behaviour of the nucleation rate curves in Figure 4 indicates a possible non-linear dependence on the temperature. This is due to the inclusion of several temperature-dependent parameters in the expression for the nucleation rate (Eq. (13)). An analytical expression for the optimal shear rate cannot be obtained because of the dependence of the exponential term and kinetic pre-factor on the shear rate.

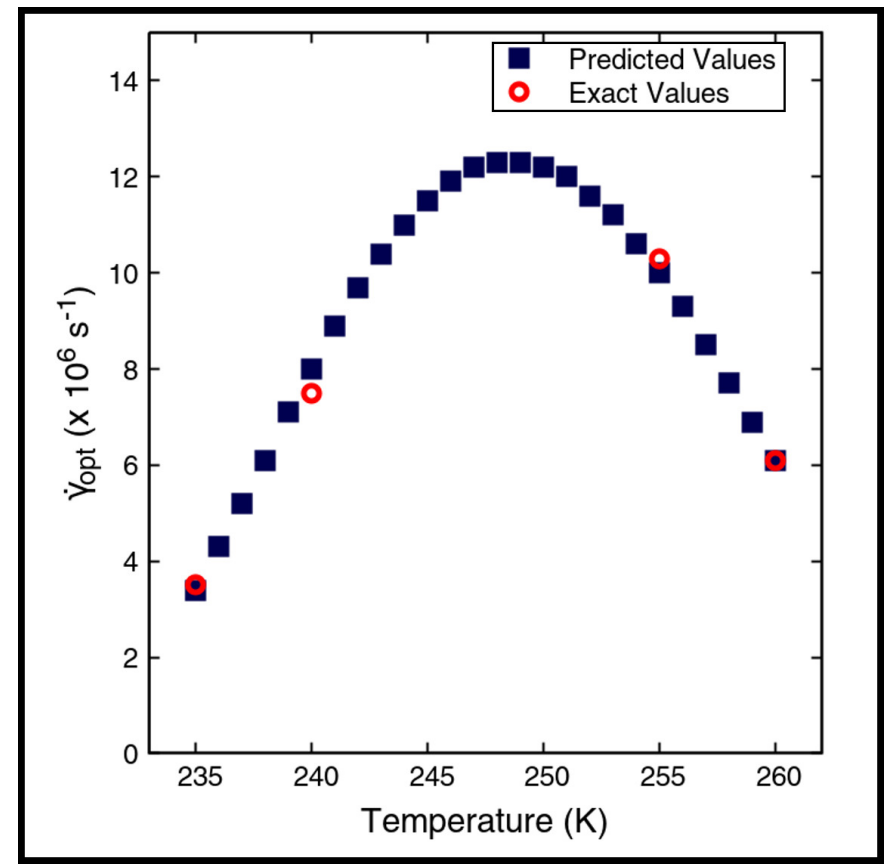

Figure 5: Temperature dependence of the predicted and exact values of the optimal shear rates $\dot{\gamma}_{o p t}$. The predicted optimal shear rates and the optimal shear rates calculated from seeded simulations are denoted by filled cobalt blue squares and open red circles, respectively. The maximum value of the optimal shear rate is observed at $248 K$. 
In order to validate the functional form of the trend from our four simulations, we employ statistical inference. We qualitatively ascertain the non-linear temperature dependence of the nucleation rates and optimal shear rates, by extrapolating data for different temperatures using the approximations outlined below. The validity of the predicted results can be assessed by comparing them with the more rigrously obtained data described in the previous section.

1. The interfacial energy $\sigma_{0}$ for all temperatures is assumed to be constant and taken to be the mean of the values calculated from seeding in Table S1. We have used this assumption with the understanding that the maximum variation in the calculated values of $\sigma_{0}$ is within $1.5 \%$, and that the statistical uncertainty in the interfacial energy calculations is $\approx 7 \%$ [28].

2. The diffusion coefficients are assumed to vary with shear according to a linear relationship given by Eq.(23), at every temperature. The values of the parameters $D_{0}$ and $c$, obtained in Section 4.1, are estimated for each temperature from linear regression. The linear fits of $D_{0}$ and $c$ with temperature are shown in Figure S6.

3. The chemical potential $\left|\Delta \mu_{0}\right|$ and the viscosity $\eta$ at each temperature are approximated by Eq. 21] and Eq. (20], respectively.

4. The density of $\mathrm{Ih}$ is assumed to be constant in the range of temperatures $235 \mathrm{~K}$ to $260 \mathrm{~K}$.

Using the approximations detailed above, we obtained the optimal shear rates for incrementally increased temperatures between $235 \mathrm{~K}$ and $260 \mathrm{~K}$. Figure 5 shows the variation of the predicted optimal shear rates with temperature. The more exact calculations for $235,240,255$ and $260 \mathrm{~K}$ are plotted alongside the predicted values, showing agreement within $10 \%$. It is evident that the predicted optimal shear rates show a non-monotonic dependence on the temperature, exhibiting a maximum at $248 \mathrm{~K}$. The values of both the predicted and exact optimal shear rates at $260 \mathrm{~K}$ are less than those at $255 \mathrm{~K}$ and $240 \mathrm{~K}$. The agreement of the predicted nucleation rate curves and the nucleation rate curves obtained in the previous section from seeding calculations is graphically shown in Figure S7.

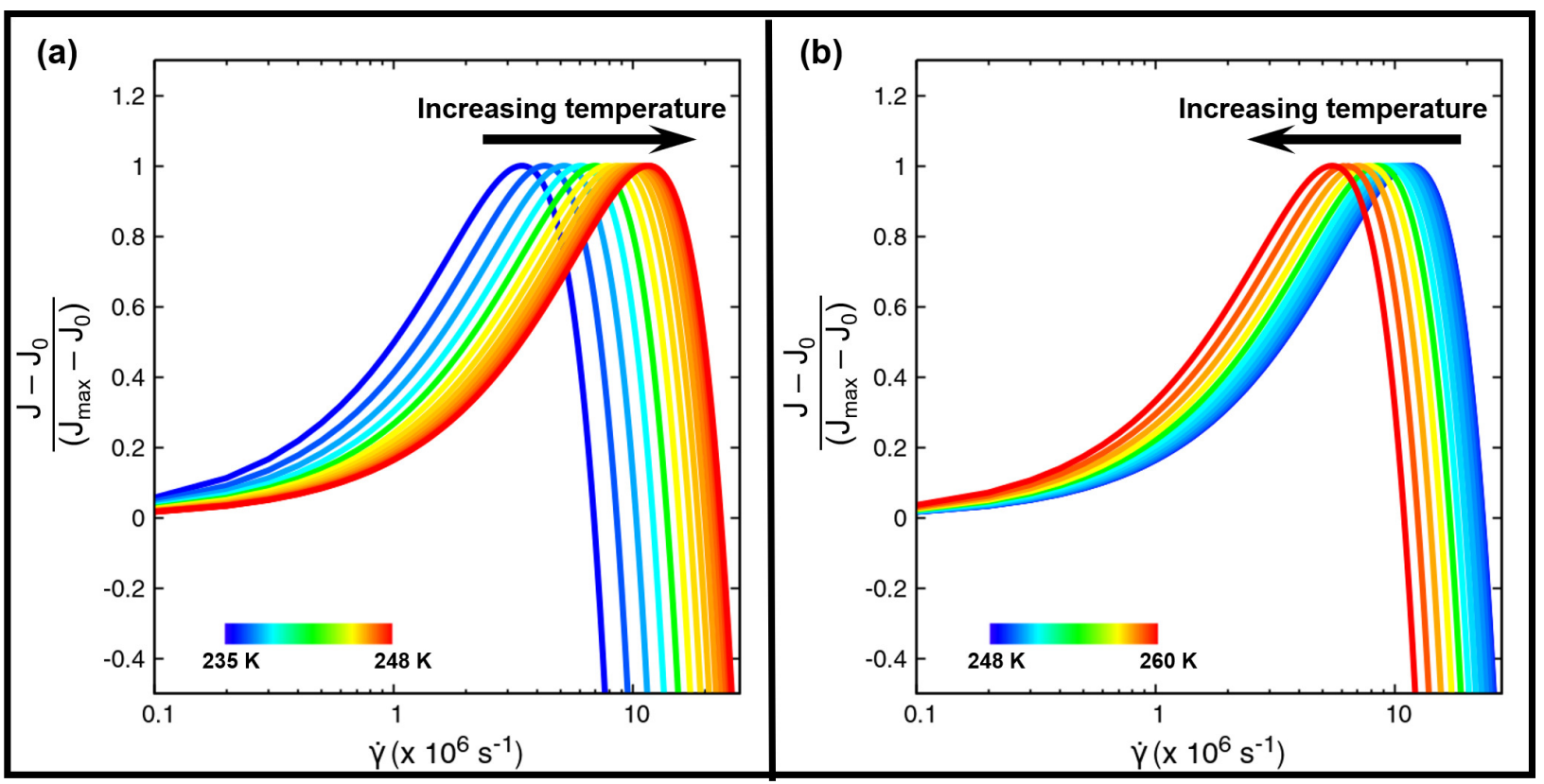

Figure 6: (a) Predicted normalized relative nucleation rate curves, varying with the shear rate $\dot{\gamma}$, for temperatures between $235 \mathrm{~K}$ and $248 \mathrm{~K}$. The optimal shear rate at each temperature increases from $235 \mathrm{~K}$ and reaches a maximum value at $248 \mathrm{~K}$. (b) Shear-dependent predicted nucleation rate curves for $248 \mathrm{~K}$ to $260 \mathrm{~K}$. Arrows signify the direction of increasing temperature.

Figure 6(a) depicts the predicted nucleation rate curves, plotted against the shear rate, for temperatures between $235 \mathrm{~K}$ and $248 \mathrm{~K}$. The nucleation rate curves are shifted in the $+x$ direction with increasing temperature. The nucleation rate curve at $248 K$ has the highest optimal shear rate and is the rightmost curve. Figure 6(b) shows the nucleation rate curves from $248 \mathrm{~K}$ to $260 \mathrm{~K}$. As the temperature increases from $248 \mathrm{~K}$, the curves are shifted in the $-x$ direction. Thus, the predicted results reinforce the suggestion of the non-monotonic temperature dependence of both the nucleation rates and the optimal shear rates. 
However, although the approximations and predicted data offer insight into the non-linear temperature dependence, there are a few unavoidable caveats. Particularly the approximation in Eq.(21), used for the chemical potential at different temperatures, is more-or-less valid over a wide range of temperatures for the $\mathrm{mW}$ water model only. Presumably, this approximation would only be acceptable for atomistic water potentials in the limit of strong supercooling. Unentangling the complex composite effects of temperature on the nucleation rate is non-trivial using more rigorous calculations, and requires further investigation.

\subsection{Dependence of the Maximum Shear Rate on Temperature}

We recall that, at a particular temperature $T$, nucleation is suppressed for shear rates greater than the maximum limiting shear rate $\dot{\gamma}_{\max }$. We can use the expression given by Eq. (22) to obtain the maximum shear rate $\dot{\gamma}_{\max }$ as a function of the temperature $T$.

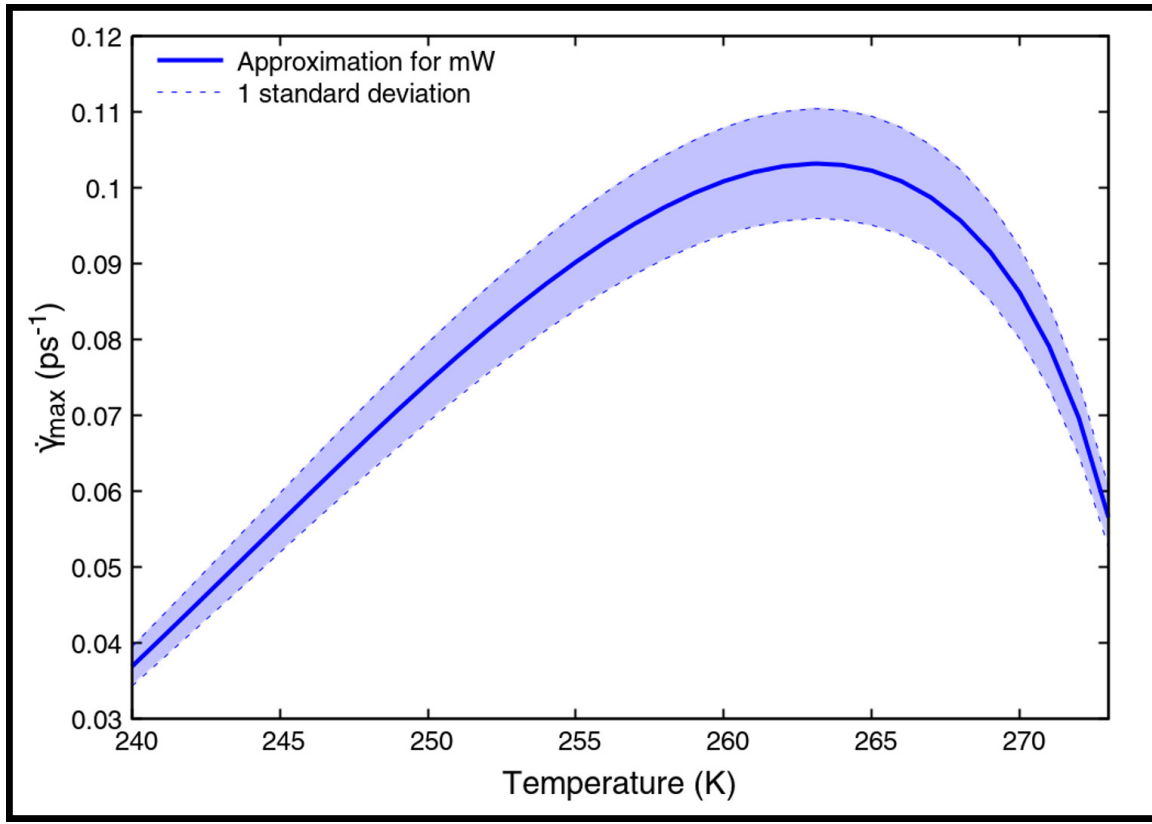

Figure 7: Maximum limiting values of the shear rate plotted against temperature for the $\mathrm{mW}$ water model, estimated using Eq.22]. The dotted blue lines denote the uncertainty in the calculation of $\dot{\gamma}_{\max }$.

Figure 7 shows the variation of $\dot{\gamma}_{\max }$ with $T$ for the $\mathrm{mW}$ water model. Eq. 22 is a smooth, continuous and differentiable function in $T$, and exhibits a maximum for a critical value of temperature, as shown in Figure 7 .

Maximizing Eq.22) with respect to $T$, we obtain a critical $T_{c}$ for which the $\dot{\gamma}_{\max }$ is maximum, given by

$$
T_{c}=\frac{2 \beta}{2 \beta+1}\left(T_{m}+T_{S}\right),
$$

where $\beta$ and $T_{S}$ are fitting parameters from Eq.20, and $T_{m}$ is the melting point.

$T_{c}$ is the temperature at which crystal nuclei survive at the highest shear rate, for a particular water model.

Thus, the maximum shear rate, $\dot{\gamma}_{c, \max }$, beyond which nucleation is suppressed at all temperatures is given by the maxima of Eq.22, and we obtain:

$$
\dot{\gamma}_{c, \max }=\frac{1}{A_{0}}\left(\frac{2 \beta}{T_{S}}\right)^{\beta}\left(\frac{T_{m}-T_{S}}{2 \beta+1}\right)^{\beta+\frac{1}{2}} \sqrt{\frac{2 G \Delta H_{m}}{v^{\prime} T_{m}}},
$$

where $A_{0}$ is a fitting parameter in Eq. 20 with the units of dynamic viscosity, and $\Delta H_{m}$ is the enthalpy change of melting. 
We note that there is no dependence of $T_{c}$ on the shear modulus $G$. This is reaffirmed by the plots of $\dot{\gamma}_{\max }$ with $T$ in Figure $\mathrm{S} 2$, wherein $T_{c}=263.2 \mathrm{~K}$ for all three shear moduli.

\section{Conclusions}

In this work, we have derived an extension to the CNT equations, explicitly accounting for shear. We have presented a biasing technique for studying the effect of shear on nucleation rates, combined with the seeding method, for studying nucleation at moderate supercooling. At such temperatures, brute-force MD simulations can be infeasible, especially since the shear rate increases the barrier height.

Our results reveal the existence of different shear regimes of nucleation behaviour for the $\mathrm{mW}$ water model: 1) low shear rates in the range of $0-1000 \mathrm{~s}^{-1}$, for which the nucleation rate is unaffected;2) high shear rates in the range of $10^{6}-10^{7} s^{-1}$, in which range a maxima in the nucleation rate is observed; 3 ) very high shear rates for which nucleation is retarded. We identify crossover shear rates indicating the increase of the nucleation rate with shear in the range of $1000-10,000 s^{-1}$. We also define optimal shear rates as those corresponding to the maximum in the nucleation rate at a particular temperature. Our formalism is thus capable of predicting trends over a wide range of shear rates, in a relatively computationally inexpensive manner. Experiments support that there is little effect on nucleation in the regime of low shear rates [15-17]. The non-monotonicity and decrease in nucleation rates due to shear agree with previous simulation studies in the literature [13, 23-26]. The non-monotonicity arises from the competing kinetic and energetic effects of shear on the nucleation rate.

The behaviour of the shear-dependent nucleation rates at each temperature hints at a possible non-linear dependence on temperature. Using various approximations, we predict that the optimal shear rates and nucleation rates have a non-monotonic dependence on temperature. We show that the predicted data agree with the results obtained from seeded simulations reasonably well enough to make definite assessments. In the future, further investigations could reveal the role of temperature in the complex interplay of factors influencing the nucleation rate.

We have calculated the analytical maximum limiting shear rate, beyond which the nucleation rate vanishes. At the maximum limiting shear rate, nucleation is completely suppressed due to the mechanical failure of incipient crystallites. We observe that the maximum limiting shear rate also has a non-monotonic dependence on temperature, similar to the optimal shear rates.

Although we have studied the $\mathrm{mW}$ water model in detail in this work, the formalism presented is general and applicable to other systems as well. By using analytical approximations within the framework of CNT, we are able to qualitatively describe the nucleation behaviour of simply sheared systems without resorting to computationally expensive simulations. Our results indicate a tantalizing, hitherto unexplored, non-monotonic temperature dependence of the shear-dependent nucleation rate curves of water. We envisage that this formalism can be used to provide insight into the qualitative nucleation behaviour of flowing systems inaccessible to brute-force MD and equilibrium rare-event acceleration techniques.

\section{Acknowledgements}

This work was supported by the Science and Engineering Research Board (sanction number STR/2019/000090 and CRG/2019/001325). Computational resources were provided by the HPC cluster of the Computer Center (CC), Indian Institute of Technology Kanpur.

\section{References}

1. Penkova, A., Pan, W., Hodjaoglu, F. \& Vekilov, P. G. Nucleation of Protein Crystals under the Influence of Solution Shear Flow. Annals of the New York Academy of Sciences 1077, 214-231 (Sept. 2006).

2. Woodhouse, F. G. \& Goldstein, R. E. Cytoplasmic streaming in plant cells emerges naturally by microfilament self-organization. Proceedings of the National Academy of Sciences 110, 14132-14137 (Aug. 2013).

3. Berland, C. R. et al. Solid-liquid phase boundaries of lens protein solutions. Proceedings of the National Academy of Sciences 89, 1214-1218 (Feb. 1992).

4. Baird, J. A., Santiago-Quinonez, D., Rinaldi, C. \& Taylor, L. S. Role of Viscosity in Influencing the Glass-Forming Ability of Organic Molecules from the Undercooled Melt State. Pharmaceutical Research 29, 271-284 (July 2011).

5. Forsyth, C. et al. Influence of Controlled Fluid Shear on Nucleation Rates in Glycine Aqueous Solutions. Crystal Growth \& Design 15, 94-102 (Nov. 2014). 
6. Ken Kelton, A. L. G. Nucleation in Condensed Matter: Applications in Materials and Biology (ed Greer, A. L.) (Elsevier, 2010).

7. Ackerson, B. J. \& Pusey, P. N. Shear-Induced Order in Suspensions of Hard Spheres. Physical Review Letters 61, 1033-1036 (Aug. 1988).

8. Yan, Y., Dhont, J., Smits, C. \& Lekkerkerker, H. Oscillatory-shear-induced order in nonaqueous dispersions of charged colloidal spheres. Physica A: Statistical Mechanics and its Applications 202, 68-80 (Jan. 1994).

9. Haw, M. D., Poon, W. C. K. \& Pusey, P. N. Direct observation of oscillatory-shear-induced order in colloidal suspensions. Physical Review E 57, 6859-6864 (June 1998).

10. Amos, R., Rarity, J., Tapster, P., Shepherd, T. \& Kitson, S. Fabrication of large-area face-centered-cubic hardsphere colloidal crystals by shear alignment. Physical Review E 61, 2929-2935 (Mar. 2000).

11. Palberg, T., Mönch, W., Schwarz, J. \& Leiderer, P. Grain size control in polycrystalline colloidal solids. The Journal of Chemical Physics 102, 5082-5087 (Mar. 1995).

12. Okubo, T. \& Ishiki, H. Kinetic Analyses of Colloidal Crystallization in a Sinusoidal Electric Field as Studied by Reflection Spectroscopy. Journal of Colloid and Interface Science 211, 151-159 (Mar. 1999).

13. Blaak, R., Auer, S., Frenkel, D. \& Löwen, H. Crystal Nucleation of Colloidal Suspensions under Shear. Physical Review Letters 93 (Aug. 2004).

14. Blaak, R., Auer, S., Frenkel, D. \& Löwen, H. Homogeneous nucleation of colloidal melts under the influence of shearing fields. Journal of Physics: Condensed Matter 16, S3873-S3884 (Sept. 2004).

15. Hansen, P. H. F., Arnebrant, T. \& Bergström, L. Shear induced aggregation of a pectin stabilised emulsion in two dimensions. Colloid \& Polymer Science 279, 153-160 (Feb. 2001).

16. Akio, S., Seiji, O., Akira, T., Hiroshi, U. \& Ken'ichi, T. Fundamental research on external factors affecting the freezing of supercooled water. International Journal of Heat and Mass Transfer 35, 2527-2536 (Oct. 1992).

17. Liu, X., Zhuang, K., Lin, S., Zhang, Z. \& Li, X. Determination of Supercooling Degree, Nucleation and Growth Rates, and Particle Size for Ice Slurry Crystallization in Vacuum. Crystals 7, 128 (May 2017).

18. Cerdà, J. J., Sintes, T., Holm, C., Sorensen, C. M. \& Chakrabarti, A. Shear effects on crystal nucleation in colloidal suspensions. Physical Review E 78 (Sept. 2008).

19. Mokshin, A. V. \& Barrat, J.-L. Shear induced structural ordering of a model metallic glass. The Journal of Chemical Physics 130, 034502 (Jan. 2009).

20. Graham, R. S. \& Olmsted, P. D. Coarse-Grained Simulations of Flow-Induced Nucleation in Semicrystalline Polymers. Physical Review Letters 103 (Sept. 2009).

21. Shao, Z. et al. Shear-accelerated crystallization in a supercooled atomic liquid. Physical Review E 91 (Feb. 2015 ).

22. Ruiz-Franco, J. et al. Crystal-to-Crystal Transition of Ultrasoft Colloids under Shear. Physical Review Letters 120 (Feb. 2018).

23. Mokshin, A. V., Galimzyanov, B. N. \& Barrat, J.-L. Extension of classical nucleation theory for uniformly sheared systems. Physical Review E 87 (June 2013).

24. Richard, D. \& Speck, T. The role of shear in crystallization kinetics: From suppression to enhancement. Scientific Reports 5 (Sept. 2015).

25. Mura, F. \& Zaccone, A. Effects of shear flow on phase nucleation and crystallization. Physical Review E 93 (Apr. 2016).

26. Luo, S., Wang, J. \& Li, Z. Homogeneous Ice Nucleation Under Shear. The Journal of Physical Chemistry B 124, 3701-3708 (Mar. 2020).

27. Sosso, G. C. et al. Crystal Nucleation in Liquids: Open Questions and Future Challenges in Molecular Dynamics Simulations. Chemical Reviews 116, 7078-7116 (May 2016).

28. Espinosa, J. R., Sanz, E., Valeriani, C. \& Vega, C. Homogeneous ice nucleation evaluated for several water models. The Journal of Chemical Physics 141, 18C529 (Nov. 2014).

29. Espinosa, J. R., Vega, C., Valeriani, C. \& Sanz, E. The crystal-fluid interfacial free energy and nucleation rate of $\mathrm{NaCl}$ from different simulation methods. The Journal of Chemical Physics 142, 194709 (May 2015).

30. Espinosa, J. R., Vega, C., Valeriani, C. \& Sanz, E. Seeding approach to crystal nucleation. The Journal of Chemical Physics 144, 034501 (Jan. 2016).

31. Volmer, M. \& Weber, A. Nucleus formation in supersaturated systems. Z. physik. Chem. 119, 277-301 (1926).

32. Becker, R. \& Döring, W. Kinetische Behandlung der Keimbildung in übersättigten Dämpfen. Annalen der Physik 416, 719-752 (1935).

33. in. Selected Works of Yakov Borisovich Zeldovich, Volume I (ed Sunyaev, R. A.) 120-137 (Princeton University Press, Dec. 1992). 
34. Bai, X.-M. \& Li, M. Test of classical nucleation theory via molecular-dynamics simulation. The Journal of Chemical Physics 122, 224510 (June 2005).

35. Bai, X.-M. \& Li, M. Calculation of solid-liquid interfacial free energy: A classical nucleation theory based approach. The Journal of Chemical Physics 124, 124707 (Mar. 2006).

36. Kelton, K. F., Greer, A. L. \& Thompson, C. V. Transient nucleation in condensed systems. The Journal of Chemical Physics 79, 6261-6276 (Dec. 1983).

37. Kelton, K. in Solid State Physics 75-177 (Elsevier, 1991).

38. Auer, S. \& Frenkel, D. Prediction of absolute crystal-nucleation rate in hard-sphere colloids. Nature 409, 10201023 (Feb. 2001).

39. Auer, S. \& Frenkel, D. Numerical prediction of absolute crystallization rates in hard-sphere colloids. The Journal of Chemical Physics 120, 3015-3029 (Feb. 2004).

40. Pan, A. C. \& Chandler, D. Dynamics of Nucleation in the Ising Model †. The Journal of Physical Chemistry B 108, 19681-19686 (Dec. 2004).

41. Moyal, J. E. Stochastic Processes and Statistical Physics. Journal of the Royal Statistical Society. Series B (Methodological) 11, 150-210 (Feb. 1949).

42. Kampen, N. V. Stochastic Processes in Physics and Chemistry (Elsevier, 1992).

43. Reguera, D., Rubi, J. M. \& Vilar, J. M. G. The Mesoscopic Dynamics of Thermodynamic Systems. The Journal of Physical Chemistry B 109, 21502-21515 (Nov. 2005).

44. Sandberg, W. \& Heyes, D. Self-diffusion in equilibrium and sheared liquid mixtures by molecular dynamics. Molecular Physics 85, 635-649 (June 1995).

45. Malandro, D. L. \& Lacks, D. J. Molecular-Level Mechanical Instabilities and Enhanced Self-Diffusion in Flowing Liquids. Physical Review Letters 81, 5576-5579 (Dec. 1998).

46. Cussler, E. Diffusion: Mass Transfer in Fluid Systems (Cambridge University Press, 2009).

47. Li, Z. Critical particle size where the Stokes-Einstein relation breaks down. Physical Review E 80 (Dec. 2009).

48. Dehaoui, A., Issenmann, B. \& Caupin, F. Viscosity of deeply supercooled water and its coupling to molecular diffusion. Proceedings of the National Academy of Sciences 112, 12020-12025 (Sept. 2015).

49. Molinero, V. \& Moore, E. B. Water Modeled As an Intermediate Element between Carbon and Silicon†. The Journal of Physical Chemistry B 113, 4008-4016 (Apr. 2009).

50. Pugliese, P., Conde, M. M., Rovere, M. \& Gallo, P. Freezing Temperatures, Ice Nanotubes Structures, and Proton Ordering of TIP4P/ICE Water inside Single Wall Carbon Nanotubes. The Journal of Physical Chemistry B 121, 10371-10381 (Nov. 2017).

51. Vega, C., Abascal, J. L. F., Conde, M. M. \& Aragones, J. L. What ice can teach us about water interactions: a critical comparison of the performance of different water models. Faraday Discuss. 141, 251-276 (2009).

52. Vega, C. \& Abascal, J. L. F. Simulating water with rigid non-polarizable models: a general perspective. Physical Chemistry Chemical Physics 13, 19663 (2011).

53. Feistel, R. \& Wagner, W. A New Equation of State for H2O Ice Ih. Journal of Physical and Chemical Reference Data 35, 1021-1047 (June 2006).

54. Vega, C. \& Noya, E. G. Revisiting the Frenkel-Ladd method to compute the free energy of solids: The Einstein molecule approach. The Journal of Chemical Physics 127, 154113 (Oct. 2007).

55. Zaragoza, A. et al. Competition between ices Ih and Ic in homogeneous water freezing. The Journal of Chemical Physics 143, 134504 (Oct. 2015).

56. De Hijes, P. M., Sanz, E., Joly, L., Valeriani, C. \& Caupin, F. Viscosity and self-diffusion of supercooled and stretched water from molecular dynamics simulations. The Journal of Chemical Physics 149, 094503 (Sept. 2018).

57. Balasubramanian, S., Mundy, C. J. \& Klein, M. L. Shear viscosity of polar fluids: Molecular dynamics calculations of water. The Journal of Chemical Physics 105, 11190-11195 (Dec. 1996).

58. Loerting, T. \& Giovambattista, N. Amorphous ices: experiments and numerical simulations. Journal of Physics: Condensed Matter 18, R919-R977 (Nov. 2006).

59. Cao, P. et al. Mechanical properties of bi- and poly-crystalline ice. AIP Advances 8, 125108 (Dec. 2018).

60. Moreira, P. A. F. P., de Aguiar Veiga, R. G. \& de Koning, M. Elastic constants of ice Ih as described by semi-empirical water models. The Journal of Chemical Physics 150, 044503 (Jan. 2019).

61. Plimpton, S. Fast Parallel Algorithms for Short-Range Molecular Dynamics. Journal of Computational Physics 117, 1-19 (Mar. 1995).

62. Maras, E., Trushin, O., Stukowski, A., Ala-Nissila, T. \& Jónsson, H. Global transition path search for dislocation formation in $\mathrm{Ge}$ on $\mathrm{Si}(001)$. Computer Physics Communications 205, 13-21 (Aug. 2016). 
63. Stukowski, A. Visualization and analysis of atomistic simulation data with OVITO-the Open Visualization Tool. Modelling and Simulation in Materials Science and Engineering 18, 015012 (Dec. 2009).

64. Goswami, R., Goswami, A. \& Singh, J. K. d-SEAMS: Deferred Structural Elucidation Analysis for Molecular Simulations. Journal of Chemical Information and Modeling 60, 2169-2177 (Mar. 2020).

65. Giorgino, T. Computing diffusion coefficients in macromolecular simulations: the Diffusion Coefficient Tool for VMD. Journal of Open Source Software 4, 1698 (Sept. 2019).

66. Evans, D. J. \& Morriss, G. P. Nonlinear-response theory for steady planar Couette flow. Physical Review A 30, 1528-1530 (Sept. 1984).

67. Lees, A. W. \& Edwards, S. F. The computer study of transport processes under extreme conditions. Journal of Physics C: Solid State Physics 5, 1921-1928 (Aug. 1972).

68. Daivis, P. J. \& Todd, B. D. A simple, direct derivation and proof of the validity of the SLLOD equations of motion for generalized homogeneous flows. The Journal of Chemical Physics 124, 194103 (May 2006).

69. Engineering Plasticity and Its Applications From Nanoscale to Macroscale Apr. 2009.

70. Kumar, V., Nazari, B., Bousfield, D. \& Toivakka, M. Rheology of microfibrillated cellulose suspensions in pressure-driven flow. eng (2016). 